\title{
Accumulation Ratio Cmax
}

National Cancer Institute

\section{Source}

National Cancer Institute. Accumulation Ratio Cmax. NCI Thesaurus. Code C102357.

The maximum concentration at steady state divided by the maximum concentration during the initial dosing interval. 